\title{
CONDITIONING REGIMEN FOR LANGERHANS HISTIOCYTOSIS
}

\author{
Victor Gottardello Zecchin ${ }^{1}$, Monica dos Santos Cypriano², Gustavo Zamperlini²
}

\author{
1 Beneficência Portuguesa de São Paulo \\ 2 Instituto de Oncologia Pediatrica - GRAACC - UNIFESP
}

Correspondence to: vgzecchin@gmail.com

Langerhans cell histiocytosis $(\mathrm{LCH})$ is a rare disease, with an estimated incidence of 0.5 per 100,000 children in the United States of America'. HCL occurs due to differentiation of myeloid precursors into CD1a+ / CD207+ cells and is characterized by constitutional activation of the MAPK2 signaling pathway, leading to a spectrum of organ involvement and dysfunction. Treatment of $\mathrm{HCL}$ is risk-adjusted: single lesions may respond to local treatment whereas multisystem disease requires systemic therapy. Although survival for patients without organ dysfunction is excellent ${ }^{3}$, mortality in those with compromised organs at risk (hematopoietic system, liver, and/or spleen) reaches $20 \%$, 2 . Despite the progress made in the treatment of $\mathrm{HCL}$, disease reactivation rates remain above $30 \%$ and the best second-line treatment has not yet been established. Treatment failure is associated with increased morbidity and mortality, including an association with neurodegeneration ${ }^{2}$.

As it is a rare disease and generally has a good prognosis, few scientific studies are evaluating the role of allogeneic hematopoietic stem cell transplantation (HSCT) in the treatment of this disease.

In 2015 Veys et $\mathrm{al}^{5}$ published retrospective results of 87 high-risk patients transplanted between 1990 and 2013. Myeloablative conditioning regimens (MAC) based on total body irradiation or busulfan ${ }^{6}$ were the most used until the 2000s, and reduced-intensity conditioning regimens (RIC) - predominantly a combination of Melphalan and Fludarabine - were most used between 2000 and 2013. Transplant-as- sociated mortality rates in 3 years were similar between RIC and MAC conditioning regimens (21\% versus $15 \%$, respectively). Recurrence was higher in the RIC group compared to the MAC group ( $28 \%$ versus $8 \%$, respectively), however, the 3-year overall survival (OS) was similar (77\% versus $71 \%$, respectively), since the patients who relapsed after RIC transplantation could be rescued with chemotherapy.

More recently, Kudo et al7 published a retrospective study with 30 patients with refractory LCH who underwent HSCT between 1996 and 2014. Eleven patients received myeloablative conditioning regimen based on total body radiotherapy (RCT) with a dose equal to or greater than $8 \mathrm{~Gy}$ or busulfan, and 19 of reduced intensity based on Fludarabine and Melphalan, associated or not with low dose of RCT. There was no significant difference between the conditioning regimen modalities, with OS of $56.8 \%$ for the RIC group and $63.6 \%$ for the MAC group. Disease status was the main prognostic factor, with a 5-year OS of $100 \%$ for patients who arrived at HSCT with disease in remission or with partial remission, versus $54.5 \%$ for those who had active disease at the time of the procedure.

Regarding the type of donor used and the source of stem cells, there is great variation, with greater frequency for unrelated and extensive use of bone marrow and umbilical cord, and apparently, there is no impact on survival rates. ${ }^{5,6}$

There are few case reports and extremely restricted performance of autologous HSCT in HCL.

\begin{tabular}{|c|c|c|c|}
\hline & Autologous & MRD & MUD \\
\hline Localized Disease & Not indicated & Not indicated & Not indicated \\
\hline Multisystem involvement & & & \\
\hline \multirow{2}{*y}{$\geq 1^{\text {a } C C R^{*}}$} & Not indicated & Standard & Standard \\
\cline { 1 - 1 } (if previously refractory) & & & \\
\hline
\end{tabular}




\section{REFERENCE}

1. Al-Ammar AY, Tewfik TL, Bond M, et al. Langerhans' cell histiocytosis: paediatric head and neck study. J Otolaryngol. 1999;28(5):266-72

2. Rodriguez-Galindo C, Allen CE. Langerhans cell histiocytosis. Blood. 2020;135(16):1319-31.

3. Morimoto A, Shioda $Y$, Imamura T, et al. Intensified and prolonged therapy comprising cytarabine, vincristine and prednisolone improves outcome in patients with multisystem Langerhans cell histiocytosis: results of the Japan Langerhans Cell Histiocytosis Study Group-02 Protocol Study. Int J Hematol. 2016;104(1):99-109.

4. Postini $A M$, del Prever AB, Pagano $M$, et al. Langerhans cell histiocytosis, 40 years' experience. J Pediatr Hematol Oncol. 2012;34(5):353-8.
5. Veys PA, Nanduri V, Baker KS, et al. Haematopoietic stem cell transplantation for refractory Langerhans cell histiocytosis: outcome by intensity of conditioning. Br J Haematol. 2015;169(5):711-8.

6. Steiner M, Matthes-Martin S, Attarbaschi A, et al. Improved outcome of treatment-resistant highrisk Langerhans cell histiocytosis after allogeneic stem cell transplantation with reduced-intensity conditioning. Bone Marrow Transplantation. 2005;36(3):215-25.

7. Kazuko Kudo, Miho Maeda, Nobuhiro Suzuki, et al. Nationwide Retrospective Review of Hematopoietic Stem Cell Transplantation in Children With Refractory Langerhans Cell Histiocytosis. Int J Hematol. 2020;111(1):137-48. 